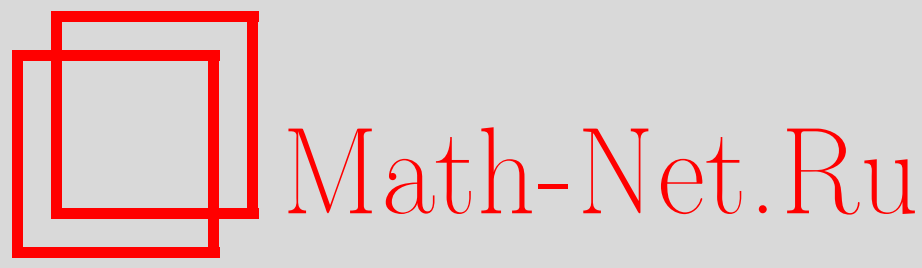

3. Коричи, М. Т. Мефтах, Изучение квантовых статистических систем в $D$-мерном пространстве с использованием дробной производной, ТМФ, 2016, том 186, номер 3, 433-442

DOI: https://doi.org/10.4213/tmf8824

Использование Общероссийского математического портала Math-Net.Ru подразумевает, что вы прочитали и согласны с пользовательским соглашением http://www . mathnet.ru/rus/agreement

Параметры загрузки:

IP : 54.237 .59 .107

26 апреля 2023 г., 15:06:34

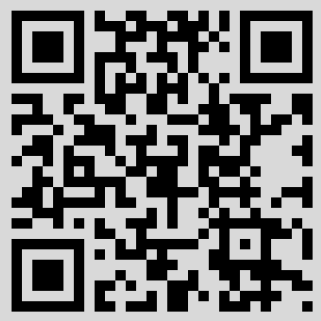




\title{
ИЗУЧЕНИЕ КВАНТОВЫХ СТАТИСТИЧЕСКИХ СИСТЕМ В D-МЕРНОМ ПРОСТРАНСТВЕ С ИСПОЛЬЗОВАНИЕМ ДРОБНОЙ ПРОИЗВОДНОЙ
}

\begin{abstract}
Изучаются термодинамические свойства некоторых квантовых статистических систем, представленных дробным гамильтонианом в $D$-мерном пространстве. Вычислены статистическая сумма системы, состоящей из $N$ дробных квантовых осцилляторов, и связанные с ней термодинамические величины. Термические и критические свойства бозе-газа и ферми-газа рассматриваются в контексте дробной энергии и описываются дробной производной.
\end{abstract}

Ключевые слова: квантовая система, статистическая сумма, дробная производная, осциллятор, бозе-система, конденсация, критическая температура, ферми-система, термодинамические свойства.

DOI: $10.4213 / \operatorname{tmf} 8824$

\section{1. ВВЕДЕНИЕ}

Дробная производная является обобщением обычной производной на случай произвольного нецелого порядка. Это понятие является давно известным, поскольку классическое исчисление целого порядка восходит к временам Ньютона и Лейбница. В своей переписке с Лейбницем Лопиталь задал ему вопрос о значении выражения $d^{n} y / d x^{n}$, если $n=1 / 2$ : “Что если $n$ является дробным числом?". Лейбниц ответил, что это приведет к парадоксу, который когда-нибудь будет иметь полезные последствия. Это письмо Лейбница рассматривается как момент рождения дробного исчисления [1], [2].

С момента своего появления дробная производная не переставала находить многочисленные применения в различных областях физики [3], включая классическую механику [4]-[6], хаотическую динамику [7], классический электромагнетизм [8], механику фрактальных сред [9], [10], физику плазмы [11], [12], физическую кинетику [13]-[15], теорию дальнодействующих взаимодействий [16], [17] и теорию переноса [18]. Недавно авторы работ [19]-[23] применили это понятие в квантовой физике и разработали новую дробную квантовую механику. Уравнение Шредингера - уравнение в частных производных целочисленного порядка. Интересно, что́ мы получим,

${ }^{*}$ University Kasdi Mehbah Ouargla, Ouargla, Algeria.

E-mail: zinebkorichid02@gmail.com, mewalid@yahoo.com 
если это уравнение будет содержать дробные производные. Результаты квантовой механики, полученные из понятия дробной производной, обсуждались некоторыми авторами [19], [21], [22]. В последние годы авторы работ [24], [25] обсуждали возможности введения этого понятия в квантовую статистическую физику и его физическую интерпретацию. Они также изучали некоторые проблемы теории квантовых статистических систем с энергетическим спектром дробно-степенного типа. Однако использование дробной производной в статистической физике не было изучено достаточно для того, чтобы показать, как скажется учет дробного гамильтониана на термодинамических свойствах некоторой квантовой статистической системы.

Цель настоящей работы заключается в том, чтобы изучить термодинамические свойства некоторых квантовых статистических систем в контексте дробного квантового гамильтониана в случае $D$-мерного пространства. Статья имеет следующую структуру. Раздел 2 посвящен определению дробного гамильтониана для $D$-мерных независимых квантовых осцилляторов. Используя канонический ансамбль, мы вычисляем статистическую сумму и некоторые термодинамические свойства для таких осцилляторов. В разделе 3 мы изучаем идеальные газы (бозе-газы и ферми-газы) в потенциальной ловушке в большом каноническом ансамбле и получаем термодинамические свойства и критические свойства. Переходя к пределу $\alpha=\nu=2$, мы вновь получаем хорошо известные результаты для стандартного случая (определенного для целочисленной производной). В разделе 4 приводятся выводы.

\section{2. ДРОБНЫЕ КВАНТОВЫЕ ОСЦИЛЛЯТОРЫ}

Дробный квантовый осциллятор, введенный Ласкиным [26], является дробной квантово-механической моделью с гамильтонианом

$$
H_{\alpha, \nu}=D_{\alpha}|p|^{\alpha}+q^{2}|x|^{\nu} \equiv D_{\alpha}\left(-\hbar^{2} \Delta\right)^{\alpha / 2}+q^{2}|x|^{\nu},
$$

где $D_{\alpha}$ - обобщенный коэффициент, физическая размерность которого [Energy $]^{1-\alpha} \times$ $[\text { Length }]^{\alpha} \times[\text { Time }]^{1-\alpha}, q^{2}-$ константа с размерностью $[\text { Energy }]^{1 / 2} \times[\text { Length }]^{-\nu / 2}$ (для $1<\alpha \leqslant 2,1<\nu \leqslant 2)$, а $\left(-\hbar^{2} \Delta\right)^{\alpha / 2}-$ квантовый дробный оператор Риза. При $\alpha=\nu=2$ соотношение (1) должно представлять гамильтониан гармонического осциллятора, тогда $D_{2}=(2 m)^{-1}, q^{2}=m \omega^{2} / 2$.

В этом разделе мы вычисляем статистическую сумму и статистические свойства системы, состоящей из $N$ независимых дробных квантовых осцилляторов, описываемых следующим гамильтонианом:

$$
\begin{aligned}
H_{\alpha, \nu} & =\sum_{i=1}^{N}\left(D_{\alpha}\left|p_{i}\right|^{\alpha}+q^{2}\left|x_{i}\right|^{\nu}\right) \equiv \\
& \equiv \sum_{i=1}^{N}\left[D_{\alpha}\left(-h^{2} \sum_{k=1}^{D} \frac{\partial^{2}}{\partial x_{k}^{2}}\right)^{\alpha / 2}+q^{2}\left(\sum_{k=1}^{D} x_{k}^{2}\right)^{\nu / 2}\right],
\end{aligned}
$$

где $p_{i}=\left(p_{1}, p_{2}, \ldots, p_{D}\right)$ и $x_{i}=\left(x_{1}, x_{2}, \ldots, x_{D}\right)-D$-мерные импульс и координата.

В случае $D$ измерений энергетические уровни дробного квантового осциллятора в полуклассической теории записываются следующим образом [27]:

$$
E_{n_{1}, \ldots, n_{D}}=a^{\sigma}\left(n_{1}+n_{2}+\cdots+n_{D}+d\right)^{\sigma},
$$


где $n_{i}=0,1,2, \ldots$ и

$$
a=\frac{\pi \hbar \nu D_{\alpha}^{1 / \alpha} q^{2 / \nu}}{2 B(1 / \nu, 1+1 / \alpha)}, \quad \sigma=\frac{\alpha \nu}{\alpha+\nu}, \quad d=\frac{D}{2},
$$

$B(x, y)$ - хорошо известная бета-функция (интеграл Эйлера первого рода). Заметим, что автор работы [27] вычислил собственную энергию только для одного измерения $(D=1)$.

Если мы описываем систему, используя канонический ансамбль, то термодинамику можно изучать исходя из статистической суммы

$$
Z_{N}=\frac{Z_{1}^{N}}{N !}
$$

где $Z_{1}$ - индивидуальная каноническая статистическая сумма,

$$
\begin{aligned}
Z_{1}=\sum_{n_{1}, \ldots, n_{D}=0}^{\infty} e^{-\beta E_{n_{1}, \ldots, n_{D}}} & =\sum_{n_{1}, \ldots, n_{D}=0}^{\infty} e^{-\beta a^{\sigma}\left(n_{1}+\cdots+n_{D}+d\right)^{\sigma}}= \\
& =\sum_{n=0}^{\infty} \frac{(n+D-1) !}{n !(D-1) !} e^{-\beta a^{\sigma}(n+d)^{\sigma}} .
\end{aligned}
$$

Последнее равенство появляется из-за вырождения собственной энергии $D$-мерного квантового осциллятора. С помощью введения $\delta$-представления $(\delta(x)$ - распределение Дирака) соотношение (6) может быть записано следующим образом:

$$
Z_{1}=\frac{1}{(D-1) !} \sum_{n=0} \frac{(n+D-1) !}{n !} \sum_{k=-\infty}^{\infty} e^{-\beta a^{\sigma} k} \delta\left(k-(n+d)^{\sigma}\right),
$$

или, эквивалентно,

$$
Z_{1}=\frac{1}{(D-1) !} \sum_{k=-\infty}^{\infty} e^{-\beta a^{\sigma} k} \frac{\Gamma\left(k^{1 / \sigma}-d+D\right)}{\Gamma\left(k^{1 / \sigma}-d+1\right)} \theta\left(k^{1 / \sigma}-d\right),
$$

где функция Хевисайда $\theta(x)$ появляется в результате суммирования по $\delta$-распределению. Оценивая функцию Хевисайда, мы получаем

$$
\begin{aligned}
Z_{1} & =\frac{1}{(D-1) !} \sum_{k=d^{\sigma}}^{\infty} e^{-\beta a^{\sigma} k} \frac{\Gamma\left(k^{1 / \sigma}-d+D\right)}{\Gamma\left(k^{1 / \sigma}-d+1\right)}= \\
& =\frac{1}{(D-1) !} \sum_{k=d^{\sigma}}^{\infty} e^{-\beta a^{\sigma} k}\left(k^{1 / \sigma}+d-1\right) \ldots\left(k^{1 / \sigma}-(d-1)\right)= \\
& =\frac{1}{(D-1) !} \sum_{m=0}^{D-1} S_{D-1}^{(m)} \sum_{k=d^{\sigma}}^{\infty} e^{-\beta a^{\sigma} k}\left(k^{1 / \sigma}+(d-1)\right)^{m},
\end{aligned}
$$

где $S_{D-1}^{(m)}-$ коэффициенты Стирлинга первого рода [28]. Чтобы оценить последнюю сумму в (9), мы используем биноминальное разложение

$$
\left(k^{1 / \sigma}+(d-1)\right)^{m}=(d-1)^{m} \sum_{p=0}^{m} \frac{C_{p}^{m}}{(d-1)^{p}}\left(k^{1 / \sigma}\right)^{p},
$$


где $C_{p}^{m}$ - биноминальные коэффициенты. Следовательно, индивидуальная статистическая сумма принимает новый вид:

$$
Z_{1}=\frac{1}{(D-1) !} \sum_{m=0}^{D-1} S_{D-1}^{(m)}(d-1)^{m} \sum_{p=0}^{m} \frac{C_{p}^{m}}{(d-1)^{p}} \sum_{k=d^{\sigma}}^{\infty} e^{-\beta a^{\sigma} k} k^{p / \sigma} .
$$

Заметим, что мы вновь получили стандартный результат для статистической суммы осциллятора в одном измерении, положив $D=1, S_{0}^{(0)}=1, C_{0}^{0}=1$ и $\sigma=1$.

Используя обозначения $B=p / \sigma$ и $b=\beta a^{\sigma}$, последнюю сумму в (11) можно записать следующим образом:

$$
\sum_{k=d^{\sigma}}^{\infty} e^{-\beta a^{\sigma} k} k^{p / \sigma}=\frac{\partial^{B}}{\partial(-b)^{B}} \sum_{k=d^{\sigma}}^{\infty} e^{-b k}=\left(\frac{\partial^{1 / \sigma}}{\partial(-b)^{1 / \sigma}}\right)^{p} \frac{e^{-b d^{\sigma}}}{1-e^{-b}},
$$

и индивидуальная статистическая сумма приобретает вид

$$
\begin{aligned}
Z_{1} & =\frac{1}{(D-1) !} \sum_{m=0}^{D-1} S_{D-1}^{(m)}(d-1)^{m} \sum_{p=0}^{m} \frac{C_{p}^{m}}{(d-1)^{p}}\left(\frac{\partial^{1 / \sigma}}{\partial(-b)^{1 / \sigma}}\right)^{p} \frac{e^{-b d^{\sigma}}}{1-e^{-b}}= \\
& =\frac{1}{(D-1) !}\left[\sum_{m=0}^{D-1} S_{D-1}^{(m)} \widehat{Y}^{m}\right] \frac{e^{-b d^{\sigma}}}{1-e^{-b}}= \\
& =\frac{1}{(D-1) !} \widehat{Y}(\widehat{Y}-1)(\widehat{Y}-2) \ldots(\widehat{Y}-D+2) \frac{e^{-b d^{\sigma}}}{1-e^{-b}},
\end{aligned}
$$

где $\widehat{Y}$ - оператор, задающийся выражением

$$
\widehat{Y}=d-1+\frac{\partial^{1 / \sigma}}{\partial(-b)^{1 / \sigma}}=\frac{D}{2}-1+\frac{\partial^{1 / \sigma}}{\partial(-b)^{1 / \sigma}} .
$$

Последнее равенство в (13) возникает благодаря разложению Стирлинга, определяемому коэффициентами $S_{D-1}^{(m)}$, которые удовлетворяют рекуррентной формуле

$$
\begin{gathered}
S_{n+1}^{(m)}=S_{n}^{(m-1)}-n S_{n}^{(m)}, \quad S_{n}^{(0)}=\delta_{n, 0}, \quad S_{n}^{(n)}=1, \quad n=0,1, \ldots, \\
S_{1}^{(1)}=1, \quad S_{2}^{(1)}=-S_{1}^{(1)}=-1 \quad \text { и т. д. }
\end{gathered}
$$

Разложения (13) вычисляются систематически следующим образом: для $D=2$

$$
\begin{aligned}
& Z_{1}=\frac{1}{(D-1) !}\left[\sum_{m=0}^{D-1} S_{D-1}^{(m)} \widehat{Y}^{m}\right] \frac{e^{-b d^{\sigma}}}{1-e^{-b}}=\left[S_{1}^{(0)}+S_{1}^{(1)} \widehat{Y}\right] \frac{e^{-b d^{\sigma}}}{1-e^{-b}} \widehat{Y} \frac{e^{-b d^{\sigma}}}{1-e^{-b}} \\
& \widehat{Y}=\frac{\partial^{s}}{\partial(-b)^{s}}, \quad s=\sigma^{-1},
\end{aligned}
$$

для $D=3$

$$
\begin{aligned}
Z_{1} & =\frac{1}{2}\left[S_{2}^{(0)}+S_{2}^{(1)} \widehat{Y}+S_{2}^{(2)} \widehat{Y}^{2}\right] \frac{e^{-b d^{\sigma}}}{1-e^{-b}}=\frac{1}{2 !} \widehat{Y}(\widehat{Y}-1) \frac{e^{-b d^{\sigma}}}{1-e^{-b}} \\
\widehat{Y} & =\frac{1}{2}+\frac{\partial^{s}}{\partial(-b)^{s}}
\end{aligned}
$$


для $D=4$

$$
\begin{aligned}
Z_{1} & =\frac{1}{3 !}\left[S_{3}^{(0)}+S_{3}^{(1)} \widehat{Y}+S_{3}^{(2)} \widehat{Y}^{2}+S_{3}^{(3)} \widehat{Y}^{3}\right] \frac{e^{-b d^{\sigma}}}{1-e^{-b}}=\frac{1}{3 !} \widehat{Y}(\widehat{Y}-1)(\widehat{Y}-2) \frac{e^{-b d^{\sigma}}}{1-e^{-b}}, \\
\widehat{Y} & =1+\frac{\partial^{s}}{\partial(-b)^{s}},
\end{aligned}
$$

и т. д. Остается обсудить дробную производную, появившуюся в этих формулах. Чтобы получить производную такого типа, мы воспользуемся формулой [2]

$$
\frac{\partial^{s}}{\partial x^{s}} e^{\lambda x} \equiv D^{s} e^{\lambda x}=x^{-s} E_{1,1-s}(\lambda x),
$$

где $E_{1,1-s}(\lambda x)$ - функция Миттаг-Леффлера.

Наконец, мы можем написать индивидуальную статистическую сумму (13) с помощью символа Похгаммера в более компактной форме:

$$
Z_{1}=\frac{(\widehat{Y}-D+2)_{D-1}}{(D-1) !} \frac{e^{-b d^{\sigma}}}{1-e^{-b}} .
$$

Теперь можно убедиться в том, что при $D=2, d=1$ и $\sigma=1, \widehat{Y}=-\partial / \partial b$ мы вновь получаем стандартный случай гармонического осциллятора в двух измерениях:

$$
Z_{1}=-\frac{\partial}{\partial b}\left(\frac{e^{-b}}{1-e^{-b}}\right)=\frac{1}{e^{b / 2}-e^{-b / 2}} \frac{1}{e^{b / 2}-e^{-b / 2}} .
$$

В случае больших измерений $(D>4)$ мы следуем той же подробной процедуре в формулах (16)-(18).

Знание статистической суммы $Z_{N}$ позволяет определить все термодинамические величины. Формально энергия Гельмгольца, внутренняя энергия и энтропия даются следующими выражениями:

$$
F=-k_{\mathrm{B}} T \ln Z_{N}, \quad U=-\frac{\partial \ln Z_{N}}{\partial \beta}, \quad S=-\frac{\partial F}{\partial T} .
$$

\section{3. КОНФАЙНМЕНТ ИДЕАЛЬНЫХ БОЗЕ-ГАЗА И ФЕРМИ-ГАЗА}

Одной из важных проблем статистической физики является проблема системы квантового газа. В настоящем разделе в контексте дробного гамильтониана в $D$-мерном пространстве мы изучаем статистические свойства идеального квантового газа, удерживаемого внешним дробным потенциалом.

3.1. Система бозонов. В $D$-мерном пространстве рассмотрим $N$ частиц идеального бозе-газа, заключенных в объеме $V$ и удерживаемых внешним дробным потенциалом $V(\vec{x})=q^{2}|\vec{x}|^{\nu}$. Мы полагаем, что спектральная энергия частицы этой системы определяется следующим образом:

$$
\varepsilon=D_{\alpha}|\vec{p}|^{\alpha}+q^{2}|\vec{x}|^{\nu} .
$$

Естественный способ рассмотрения этой системы (системы бозонов) заключается в использовании большой канонической статистичекской суммы $D(z, V, T)$ [29], задаваемой выражением

$$
D(z, V, T)=\prod_{k} \frac{1}{1-z e^{-\beta \varepsilon_{k}}}
$$


где $\varepsilon_{k}$ - энергия $k$-го одночастичного состояния, $z$-летучесть газа, которая связана с химическим потенциалом $\mu$ с помощью формулы

$$
z=e^{\beta \mu} .
$$

Заметим, что с помощью соотношений (23) и (24) большая каноническая статистическая сумма может быть записана следующим образом:

$$
D(z, V, T)=\prod_{\alpha} \frac{1}{1-z e^{-\beta\left(D_{\alpha}|p|^{\alpha}+q^{2}|x|^{\nu}\right)}} .
$$

Согласно (26) все термодинамические величины системы могут быть получены непосредственно.

3.1.1. Уравнения состояния. Для большого канонического ансамбля равновесной статистической механики уравнения состояния имеют вид

$$
\begin{aligned}
\frac{P V}{K T} & =\ln (D(z, V, T))=-\sum_{\alpha} \ln \left(1-z e^{-\beta\left(D_{\alpha}|p|^{\alpha}+q^{2}|x|^{\nu}\right)}\right)-\ln (1-z), \\
N & =z \frac{\partial}{\partial z} \ln (D(z, V, T))=\sum_{\alpha} \frac{z e^{-\beta\left(D_{\alpha}|p|^{\alpha}+q^{2}|x|^{\nu}\right)}}{1-z e^{-\beta\left(D_{\alpha}|p|^{\alpha}+q^{2}|x|^{\nu}\right)}}+\frac{z}{1-z},
\end{aligned}
$$

где $P$ и $N$ - давление и полное среднее число частиц в системе соответственно. В термодинамическом пределе они могут быть записаны следующим образом:

$$
\begin{aligned}
\frac{P V}{K T} & =\frac{-4 \pi^{D}}{\Gamma^{2}(D / 2) h^{D}} \int p^{D-1} d p \int_{0}^{\infty} x^{D-1} \ln \left(1-z e^{-\beta\left(D_{\alpha} p^{\alpha}+q^{2} x^{\nu}\right)}\right) d x-\ln (1-z), \\
N & =\frac{4 \pi^{D}}{\Gamma^{2}(D / 2) h^{D}} \int p^{D-1} d p \int_{0}^{\infty} x^{D-1} \frac{z e^{-\beta\left(D_{\alpha} p^{\alpha}+q^{2}|x|^{\nu}\right)}}{1-z e^{-\beta\left(D_{\alpha} p^{\alpha}+q^{2} x^{\nu}\right)}} d x+\frac{z}{1-z} .
\end{aligned}
$$

Вычисляя эти интегралы, мы окончательно получаем

$$
\begin{aligned}
\frac{P V}{K T} & =\frac{4 \pi^{D} \Gamma(D / \alpha) \Gamma(D / \nu)}{\Gamma^{2}(D / 2) h^{D} \nu \alpha(\beta)^{D / \nu+D / \alpha} q^{2 D / \nu} D_{\alpha}^{D / \alpha}} g_{D / \alpha+D / \nu+1}(z)-\ln (1-z), \\
N & =\frac{4 \pi^{D} \Gamma(D / \alpha) \Gamma(D / \nu)}{\Gamma^{2}(D / 2) h^{D} \nu \alpha(\beta)^{D / \nu+D / \alpha} q^{2 D / \nu} D_{\alpha}^{D / \alpha}} g_{D / \alpha+D / \nu}(z)+\frac{z}{1-z},
\end{aligned}
$$

где $g_{l}(z)=\sum_{k=1}^{\infty} z^{k} / k^{l}-$ функция Бозе.

$\mathrm{C}$ помощью введения параметров (виртуальное измерение)

$$
D^{\prime}=D+\frac{D \alpha}{\nu}
$$

и

$$
V^{*}=\left(\frac{D_{\alpha} h^{\alpha}}{q^{2}\left(\frac{2 \pi^{D / 2} \Gamma(D / \alpha)}{\alpha \Gamma(D / 2)}\right)^{\alpha / D}}\right)^{D / \nu} \frac{2 \pi^{D / 2} \Gamma(D / \nu+1)}{D \Gamma(D / 2)}
$$

система (28) может быть записана следующим образом:

$$
\begin{aligned}
\frac{P V}{K T} & =\frac{V^{*}}{\lambda_{g}^{D^{\prime}}} g_{D^{\prime} / \alpha+1}(z)-\ln (1-z), \\
N & =\frac{V^{*}}{\lambda_{g}^{D^{\prime}}} g_{D^{\prime} / \alpha}(z)+\frac{z}{1-z},
\end{aligned}
$$


где

$$
\lambda_{g}=\left(\frac{\Gamma(D / 2) h^{D} \alpha\left(\beta D_{\alpha}\right)^{D / \alpha}}{2 \pi^{D / 2} \Gamma(D / \alpha)}\right)^{1 / D}
$$

- обобщенная термическая длина волны [30].

Сравнение системы уравнений (29) с уравнением состояния свободного идеального бозе-газа [24]

$$
\begin{aligned}
\frac{P V}{K T} & =\frac{V}{\lambda_{g}^{D}} g_{D / \alpha+1}(z)-\ln (1-z), \\
N & =\frac{V}{\lambda_{g}^{D}} g_{D / \alpha}(z)+\frac{z}{1-z}
\end{aligned}
$$

показывает, что уравнение состояния, описывающее удерживаемые бозоны, имеет вид, аналогичный уравнению свободного бозе-газа, когда $D^{\prime}=D+D \alpha / \nu$ - размерность пространства, $V^{*}$ - объем. В этом смысле можно сказать, что удерживаемая бозе-система эквивалентна свободной бозе-системе [31].

3.1.2. Температура перехода. По аналогии со свободным газом максимальное число частиц на возбужденных уровнях может быть получено исходя из предела $z \downarrow 1$, так что мы получаем

$$
N_{\varepsilon>0}^{\max }=\frac{4 \pi^{D} \Gamma(D / \alpha) \Gamma(D / \nu)}{\Gamma^{2}(D / 2) h^{D} \nu \alpha(\beta)^{D / \nu+D / \alpha} q^{2 D / \nu} D_{\alpha}^{D / \alpha}} g_{D / \alpha+D / \nu}(1)=\frac{V^{*}}{\lambda_{g}^{D^{\prime}}} g_{D^{\prime} / \alpha}(1) .
$$

Отсюда получаем критическую температуру

$$
T_{\mathrm{B}}=\frac{1}{k_{\mathrm{B}}}\left(\frac{\nu \alpha \Gamma^{2}(D / 2) h^{D} q^{2 D / \nu} D_{\alpha}^{D / \alpha} N}{4 \pi^{D} \Gamma(D / \alpha) \Gamma(D / \nu) g_{D / \alpha+D / \nu}(1)}\right)^{\alpha \nu / D(\alpha+\nu)},
$$

а температурная зависимость фракции конденсата имеет вид

$$
N_{0}=N\left(1-\left(\frac{T}{T_{\mathrm{B}}}\right)^{D(\alpha+\nu) / \alpha \nu}\right) .
$$

3.1.3. Термодинамические свойства. Используя статистические выражения термодинамических величин, можно без труда получить термодинамические величины системы из соотношения (26). Например, внутренняя энергия этой системы записывается следующим образом:

$$
\begin{aligned}
U & =-\frac{\partial \ln (D(z, V, T))}{\partial \beta}= \\
& = \begin{cases}\left(\frac{D}{\nu}+\frac{D}{\alpha}\right) N k_{\mathrm{B}} T \frac{g_{D / \alpha+D / \nu+1}(z)}{g_{D / \alpha+D / \nu}(z)}, & T>T_{\mathrm{B}}, \\
N K T_{\mathrm{B}}\left(\frac{D}{\alpha}+\frac{D}{\nu}\right) \frac{g_{D / \alpha+D / \nu+1}(1)}{g_{D / \alpha+D / \nu}(1)}\left(\frac{T}{T_{\mathrm{B}}}\right)^{D / \alpha+D / \nu+1}, & T \leqslant T_{\mathrm{B}},\end{cases}
\end{aligned}
$$

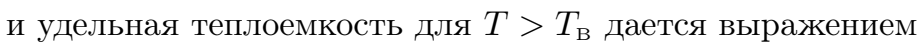

$$
\begin{aligned}
\frac{C_{V}}{N k_{\mathrm{B}}}= & \left(\frac{D}{\alpha}+\frac{D}{\nu}\right)\left(\left(\frac{D}{\alpha}+\frac{D}{\nu}\right)+1\right) \frac{g_{D / \alpha+D / \nu+1}(z)}{g_{D / \alpha+D / \nu}(z)}- \\
& -\left(\frac{D}{\alpha}+\frac{D}{\nu}\right)^{2} \frac{g_{D / \alpha+D / \nu}(z)}{g_{D / \alpha+D / \nu-1}(z)},
\end{aligned}
$$


а для $T \leqslant T_{\mathrm{B}}$

$$
\frac{C_{V}}{N k_{\mathrm{B}}}=\left(\frac{D}{\alpha}+\frac{D}{\nu}\right)\left(\left(\frac{D}{\alpha}+\frac{D}{\nu}\right)+1\right) \frac{g_{D / \alpha+D / \nu+1}(1)}{g_{D / \alpha+D / \nu}(1)}\left(\frac{T}{T_{\mathrm{B}}}\right)^{D / \alpha+D / \nu} .
$$

Энергия Гельмгольца записывается как

$$
F=N \mu-P V= \begin{cases}N k_{\mathrm{B}} T\left(\ln z-\frac{g_{D / \alpha+D / \nu+1}(z)}{g_{D / \alpha+D / \nu}(z)}\right), & T>T_{\mathrm{B}}, \\ -N k_{\mathrm{B}} T_{\mathrm{B}} \frac{g_{D / \alpha+D / \nu+1}(1)}{g_{D / \alpha+D / \nu}(1)}\left(\frac{T}{T_{\mathrm{B}}}\right)^{D / \alpha+D / \nu+1}, & T \leqslant T_{\mathrm{B}},\end{cases}
$$

и энтропия системы бозонов дается выражением

$$
S=\frac{U-F}{T}= \begin{cases}N k_{\mathrm{B}}\left(\left(\frac{D}{\alpha}+\frac{D}{\nu}+1\right) \frac{g_{D / \alpha+D / \nu+1}(z)}{g_{D / \alpha+D / \nu}(z)}-\ln z\right), & T>T_{\mathrm{B}}, \\ N k_{\mathrm{B}}\left(\frac{D}{\alpha}+\frac{D}{\nu}+1\right) \frac{g_{D / \alpha+D / \nu+1}(1)}{g_{D / \alpha+D / \nu}(1)}\left(\frac{T}{T_{\mathrm{B}}}\right)^{D / \alpha+D / \nu}, & T \leqslant T_{\mathrm{B}} .\end{cases}
$$

Если положить $\alpha=2$, то мы приходим к результатам, полученным в работе [32]. С этой точки зрения наша работа является обобщением этой работы для степенных потенциалов и импульса в выражении для гамильтониана.

3.2. Система фермионов. В случае ферми-газа большая статистическая сумма дается выражением

$$
D(z, V, T)=\prod_{k}\left(1+z e^{\left.-\beta \varepsilon_{k}\right)}\right)=\prod_{\alpha}\left(1+z e^{-\beta\left(D_{\alpha}|p|^{\alpha}+q^{2}|x|^{\nu}\right)}\right) .
$$

Отсюда следует, что давление газа и среднее число частиц $N$ могут быть записаны следующим образом:

$$
\begin{aligned}
\frac{P V}{k_{\mathrm{B}} T} & =\ln D(z, V, T)=\sum_{\alpha} \ln \left(1+z e^{-\beta\left(D_{\alpha}|p|^{\alpha}+q^{2}|x|^{\nu}\right)}\right), \\
N & =z \frac{\partial}{\partial z} \ln D(z, V, T)=\sum_{\alpha} \frac{z e^{-\beta\left(D_{\alpha}|p|^{\alpha}+q^{2}|x|^{\nu}\right)}}{1+z e^{-\beta\left(D_{\alpha}|p|^{\alpha}+q^{2}|x|^{\nu}\right)}} .
\end{aligned}
$$

Используя тот же метод, что и в случае бозе-газа, мы записываем уравнения состояния следующим образом:

$$
\begin{aligned}
\frac{P V}{K T} & =\frac{4 \pi^{D} \Gamma(D / \alpha) \Gamma(D / \nu)}{\Gamma^{2}(D / 2) h^{D} \nu \alpha(\beta)^{D / \nu+D / \alpha} q^{2 D / \nu} D_{\alpha}^{D / \alpha}} f_{D / \alpha+D / \nu+1}(z), \\
N & =\frac{4 \pi^{D} \Gamma(D / \alpha) \Gamma(D / \nu)}{\Gamma^{2}(D / 2) h^{D} \nu \alpha(\beta)^{D / \nu+D / \alpha} q^{2 D / \nu} D_{\alpha}^{D / \alpha}} f_{D / \alpha+D / \nu}(z),
\end{aligned}
$$

где $f^{l}(z)=\sum_{k=1}^{\infty}(-1)^{k+1}\left(z^{k} / k^{l}\right)-$ функция Ферми.

Все термодинамические свойства можно найти без труда: внутренняя энергия

$$
U=\left(\frac{D}{\alpha}+\frac{D}{\nu}\right) N k_{\mathrm{B}} T \frac{f_{D / \alpha+D / \nu+1}(z)}{f_{D / \alpha+D / \nu}(z)},
$$


удельная теплоемкость для постоянного объема

$$
\begin{aligned}
\frac{C_{V}}{N k_{\mathrm{B}}}= & \left(\frac{D}{\alpha}+\frac{D}{\nu}\right)\left(\left(\frac{D}{\alpha}+\frac{D}{\nu}\right)+1\right) \frac{f_{D / \alpha+D / \nu+1}(z)}{f_{D / \alpha+D / \nu}(z)}- \\
& -\left(\frac{D}{\alpha}+\frac{D}{\nu}\right)^{2} \frac{f_{D / \alpha+D / \nu}(z)}{f_{D / \alpha+D / \nu-1}(z)},
\end{aligned}
$$

энергия Гельмгольца

$$
F=N k_{\mathrm{B}} T\left(\ln z-\frac{f_{D / \alpha+D / \nu+1}(z)}{f_{D / \alpha+D / \nu}(z)}\right)
$$

энтропия

$$
S=N k_{\mathrm{B}}\left(\left(\frac{D}{\alpha}+\frac{D}{\nu}+1\right) \frac{f_{D / \alpha+D / \nu+1}(z)}{f_{D / \alpha+D / \nu}(z)}-\ln z\right) .
$$

Замечание, касающееся эквивалентности между ферми-газом в потенциальной ловушке и свободным ферми-газом, справедливо и в этом случае. Также, если мы положим $\alpha=2, \nu=2$, мы вновь получаем все стандартные результаты.

Кстати, авторы работы [33] рассматривали идеальный газ в потенциальной ловушке, экстраполируя пространственное измерение с двух до любого другого измерения. Наша работа выполнена в рамках дробной квантовой механики [19], [26], [27]. Мы также показали, что наша система с идеальным газом в потенциальной ловушке эквивалентна системе свободных бозонов; это задача, которая не рассматривается в работе [33].

\section{4. ЗАКЛЮЧЕНИЕ}

В настоящей работе мы применили дробное исчисление к статистической механике в $D$-мерном пространстве. Мы сосредоточили наше внимание на том, чтобы показать, как меняются термодинамические свойства некоторой статистической системы, описываемой дробным гамильтонианом. Другой интересный и фундаментальный вопрос заключается в том, чтобы вновь получить стандартные результаты статистической механики при переходе от дробных показателей к целым. Действительно, мы обнаружили, что статистическая сумма системы, состоящей из $N$ дробных квантовых осцилляторов, модифицирована и термодинамические свойства этой системы также меняются. Для подтверждения справедливости наших вычислений мы сравнили наши результаты с результатами в стандартном случае. Это мы проделали, сделав дробные показатели целыми $(\alpha=\nu=2)$. Еще одна система была нам интересна, а именно квантовый газ Бозе-Эйнштейна. В частности, мы исследовали проблему конденсации, которая является особым свойством этого газа. Мы обнаружили, что газ в потенциальной ловушке испытывает фазовый переход второго

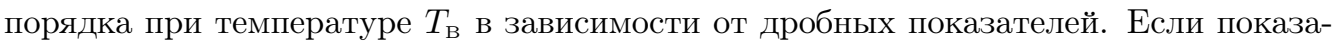
тели целые, то мы получаем стандартный случай. Такой же подход был применен к другой фундаментальной системе - к квантовому газу Ферми-Дирака. В этом случае мы также показали, что квантовый газ в потенциальной ловушке эквивалентен свободному квантовому газу. 
Благодарности. Авторы выражают признательность лаборатории LRPPS и ее директору за поддержку, содействие и некоторые предоставленные технические материалы для проведения этой работы. Мы также благодарим Prof. M. Naoua (English Department, El-Oued University) за помощь в улучшении английского языка этой статьи. Особую благодарность мы выражаем Prof. E. Koelink (Mathematics Department, Radbound University, Nijmegen, Netherlands), который сообщил нам некоторые подробности, касающиеся коэффициентов Стирлинга.

\section{Список литературы}

[1] K. S. Miller, B. Ross, An Introcduction to the Fractional Calculus and Fractional Differential Equations, John Wiley and Sons, New York, 1993.

[2] I. Podlubny, Fractional Differential Equations. An Introduction to Fractional Derivatives, Fractional Differential Equations, to Methods of Their Solution and Some of Their Applications, Mathematics in Science and Engineering, 198, Academic Press, San Diego, CA, 1999.

[3] R. Hilfer (ed.), Applications of Fractional Calculus in Physics, World Sci., Singapore, 2000.

[4] K. S. Fa, Physica A, 350:2 (2005), 199-206.

[5] F. Riewe, Phys. Rev. E, 53:2 (1996), 1890-1899.

[6] F. Riewe, Phys. Rev. E, 55:3 (1997), 3581-3592.

[7] G. M. Zaslavsky, Hamiltonian Chaos and Fractional Dynamics, Oxford Univ. Press, Oxford, 2005.

[8] A. Hussain, S. Ishfaq, Q. A. Naqvi, Prog. Electromagn. Res., 63 (2006), 319-335.

[9] V.E. Tarasov, Phys. Lett. A, 336:2-3 (2005), 167-174.

[10] V.E. Tarasov, Chaos, 15:2 (2005), 023102, 8 pp., arXiv: nlin/0602029.

[11] B. A. Carreras, V. E. Lynch, G. M. Zaslavsky, Phys. Plasmas, 8:12 (2001), 5096-5103.

[12] V.E. Tarasov, Phys. Plasmas, 12:8 (2005), 082106, 9 pp.

[13] G. M. Zaslavsky, Physica D, 76:1-3 (1994), 110-122.

[14] R. R. Nigmatullin, Physica A, 363:2 (2006), 282-298.

[15] G. M. Zaslavsky, M. A. Edelman, Physica D, 193:1-4 (2004), 128-147.

[16] N. Laskin, G. M. Zaslavsky, Physica A, 368:1 (2006), 38-54, arXiv: nlin/0512010.

[17] V.E. Tarasov, G. M. Zaslavsky, Chaos, 16:2 (2006), 023110, 13 pp.

[18] В. В. Учайкин, УФН, 173:8 (2003), 847-876.

[19] N. Laskin, Phys. Lett. A, 268:4-6 (2000), 298-305, arXiv: hep-ph/9910419; Phys. Rev. E, 62:3 (2000), 3135-3145, arXiv: 0811.1769.

[20] X. Guo, M. Xu, J. Math. Phys., 47:8 (2006), 082104, 9 pp.

[21] J. Dong, M. Xu, J. Math. Phys., 48:7 (2007), 072105, 14 pp.

[22] R. Herrmann, Gam. Ori. Chron. Phys., 1:1 (2013), 1-12, arXiv: 1210.4410.

[23] R. Herrmann, Cent. Eur. J. Phys., 11:10 (2013), 1212-1220, arXiv: 1308.4587.

[24] Z. Korichi, M. T. Meftah, J. Math. Phys., 55:3 (2014), 033302, 9 pp.

[25] 3. З. Алисултанов, Р. П. Мейланов, ТМФ, 171:3 (2012), 404-416.

[26] N. Laskin, Phys. Rev. E, 66:5 (2002), 056108, 7 pp., arXiv: quant-ph/0206098.

[27] N. Laskin, Principles of fractional quantum mechanics, arXiv: 1009.5533.

[28] М. Абрамовиц, И. Стиган (ред.), Справочник по специальным функциям с формулами, графиками и математическими таблицами, Наука, М., 1979.

[29] K. Huang, Statistical Mechanics, John Wiley and Sons, New York, 1963.

[30] Z. Yan, Eur. J. Phys., 21:6 (2000), 625-631.

[31] G. Su, Y. Cai, J. Chen, J. Phys. A: Math. Theor., 42:12 (2009), 125003, 7 pp.

[32] S. Luca, J. Math. Phys., 41:12 (2000), 8016-8024.

[33] Z. Yan, M. Li, L. Chen, C. Chen, J. Chen, J. Phys. A: Math. Gen., 32:22 (1999), 4069-4078. 